\title{
LETTERS
}

\section{Key considerations when comparing outcomes by mode of delivery raise questions about study validity and clinical relevance}

In their CMAJ article, Korb and colleagues ${ }^{1}$ investigated the important and complex issue of cesarean delivery and subsequent severe acute maternal morbidity (SAMM). Observational studies comparing maternal and perinatal outcomes following vaginal and cesarean delivery have been criticized for inappropriate comparison groups and the inability to address confounding by indication. Despite efforts to overcome these challenges, the study by Korb and colleagues ${ }^{1}$ is compromised by these same issues and, consequently, the results need to be interpreted with caution.

First, comparing outcomes following successful vaginal and cesarean deliveries is misleading with respect to causal inference. Risks of an outcome across 2 interventions can be contrasted meaningfully only if those who received one intervention were candidates for receiving the other intervention. This is known as the principle of exchangeability, ${ }^{2}$ which dictates that both groups should be exchangeable with respect to an outcome had they been subjected to the identical exposure. ${ }^{3}$ However, a successful vaginal delivery is impossible for a substantial proportion of women who deliver by cesarean. To address this limitation, in 2006 the National Institutes of Health State-of-the-Science Conference Statement on cesarean delivery recommended that all future research comparing maternal and neonatal outcomes following vaginal and cesarean delivery be based on planned vaginal versus planned cesarean delivery. ${ }^{4}$ Korb and colleagues ${ }^{1}$ applied this approach in a sensitivity analysis (Appendix $7^{1}$ ); however, the absence of a significant association between cesarean delivery and SAMM among the 3 subgroups of women aged less than $25,25-29$ and $30-34$ years (80\% of the study population) was not discussed. Instead, the authors emphasized the finding of increased SAMM following planned cesarean delivery among older women (those aged 35 years and older). Nevertheless, there was no information describing the subgroups of women with respect to key demographic and clinical characteristics (e.g., parity and body mass index) or whether women may have had a specific reason for planning a cesarean versus vaginal delivery, and this makes the validity and generalizability of the subgroup analysis unclear.

Second, although this study attempted to address confounding by indication with propensity score analysis, the indications for intervention were not quantified and, hence, not included in the propensity score. For example, no distinction was made for emergency cesarean delivery, which is invariably unplanned and therefore indicated owing to fetal or maternal reasons. In fact, all intrapartum cesarean deliveries are unplanned and indeed indicated. Furthermore, it is plausible that this unmeasured confounding by indication increased with maternal age and manifested more prominently in older women.

A clearly formulated research question and a sound understanding of confounding and the temporal sequence between exposure, outcome and factors in the causal pathway are crucial for assessing causal associations. Unfortunately, since the study by Korb and colleagues ${ }^{1}$ did not satisfy these research imperatives, the clinical relevance of their results is questionable.

Providers of maternity care should inform women about the maternal and perinatal risks associated with both cesarean and vaginal delivery, and ultimately, the balance between these risks will dictate the use of obstetric interventions.

\section{Giulia M. Muraca PhD}

Postdoctoral fellow, Clinical

Epidemiology Unit, Department of Medicine, Solna, Karolinska Institutet, Stockholm, Sweden

\section{Neda Razaz PhD}

Postdoctoral fellow, Clinical

Epidemiology Unit, Department of Medicine, Solna, Karolinska Institutet, Stockholm, Sweden

Cite as: CMAJ 2019 August 19;191:E923. doi: $10.1503 / \mathrm{cmaj} .72687$

\section{References}

1. Korb D, Goffinet F, Seco A, et al. Risk of severe maternal morbidity associated with cesarean delivery and the role of maternal age: a populationbased propensity score analysis. CMAJ 2019;191: E352-60.

2. Greenland S, Robins JM. Identifiability, exchangeability and confounding revisited. Epidemiol Perspect Innov 2009;6:4.

3. Hernán MA. A definition of causal effect for epidemiological research. J Epidemiol Community Health 2004;58:265-71.

4. NIH state-of-the-science conference statement: cesarean delivery on maternal request March 27-29, 2006. Obstet Gynecol 2006;107:1386-97.

Competing interests: None declared. 